\title{
Impact of a Severe Rocky Mountain Spotted Fever Case on Treatment Practices at an Academic Institution Within a Nonendemic Area
}

\author{
Conrad Krawiec, $\mathrm{MD}^{1}$; Gary D. Ceneviva, $\mathrm{MD}^{1}$; Shouhao Zhou, $\mathrm{PhD}^{2}$; Neal J. Thomas, $\mathrm{MD}^{1,2}$ \\ ${ }^{1}$ Pediatric Critical Care Medicine, Department of Pediatrics, Penn State Health Children's Hospital, Hershey, Pennsylvania; ${ }^{2}$ Department of Public \\ Health Sciences, Pennsylvania State University College of Medicine, Hershey, Pennsylvania
}

\begin{abstract}
Introduction-Rocky Mountain spotted fever (RMSF) is a bacterial disease associated with morbidity and mortality when untreated. The primary study objectives are to describe clinician diagnostic and treatment practices in a nonendemic area after the occurrence of an unrecognized severe pediatric presumed RMSF case (index case). We hypothesized that inpatient diagnostic testing frequency and initiation of empiric treatment will increase after the index case.

Methods-We performed a retrospective chart review of patients aged less than $18 \mathrm{y}$ evaluated for RMSF at Penn State Hershey Children's Hospital between 2010 and 2019. We divided the study population into 2 groups (preindex and postindex) and evaluated patient characteristics, RMSF testing completion, and timing of doxycycline administration.

Results-Fifty-four patients (14 [26\%] preindex and 40 [74\%] postindex) were included. Age (median $\left[25^{\text {th }}\right.$ percentile, $75^{\text {th }}$ percentile]) decreased from $14.5 \mathrm{y}(8.6,16)$ preindex to $8.3 \mathrm{y}(3.6,14)$ postindex. Twelve (86\%) preindex and $31(78 \%)$ postindex patients received empiric doxycycline $(P=0.70)$. Four years after the index case, a decrease in empiric and urgent initiation of doxycycline administration was noted. One case of severe RMSF was diagnosed 4 y after the index case.

Conclusions-Our study found that inpatient RMSF testing increased after the index case, but not all patients received empiric treatment. This may represent an underappreciation of RMSF severity even after a recent devastating case. We suggest that when severe rare but possibly reversible diseases, such as RMSF occur, all clinicians are educated on the diagnostic and treatment approach to reduce the morbidity and mortality risk.
\end{abstract}

Keywords: Rickettsia rickettsii, pediatrics, United States, tickborne illness

\section{Introduction}

Rocky Mountain spotted fever (RMSF) is a severe tickborne illness caused by the bacteria Rickettsia rickettsii. ${ }^{1}$ The common symptoms of this disease include fever, headache, myalgia, and various types of rashes (maculopapular to petechial). ${ }^{2}$ These nonspecific symptoms, along with a rash that may not be present in all cases, can result in underrecognition. ${ }^{1}$

Corresponding author: Conrad Krawiec, MD, Department of Pediatrics, Penn State Health Children's Hospital, 500 University Drive, P.O. Box 850, Hershey, PA 17033-0850; e-mail: ckrawiec@pennstatehealth.psu.edu.

Submitted for publication December 2020.

Accepted for publication May 2021.
When RMSF is not promptly recognized and treated, it can result in multiorgan system failure, including encephalopathy, acute respiratory failure due to acute respiratory distress syndrome, and septic shock. ${ }^{2-6}$ Multiple studies have demonstrated that if doxycycline administration does not occur within $5 \mathrm{~d}$ of onset of symptoms, patients are at high risk of these complications, including death. ${ }^{2,7}$ The standard of care is to initiate therapy early, even if an alternative diagnosis is more likely or the classic findings of RMSF are absent. $^{2,8}$ Thus, when a pediatrician works in an RMSF endemic area, a high index of suspicion is required; when suspected, testing and early treatment are necessary.

RMSF is commonly diagnosed in areas of the southeastern and southcentral United States, where climate and 
host availability are favorable to the various tick vectors that harbor Rickettsia. ${ }^{4,9}$ In recent years, however, there are concerns that these environmental risk factors may change due to climate change. ${ }^{10}$ This has resulted in an increase in tick bites for not only RMSF but other tickborne illnesses as well. ${ }^{10,11}$ If climate change continues, the frequency of tick bites will likely continue to rise in endemic areas, and ticks may migrate northward, placing nonendemic areas at risk. ${ }^{10,12}$

If a severe case of RMSF occurs in a nonendemic area and is unrecognized, its individual emotional impact can be profound, especially if it results in patient harm. ${ }^{13}$ This can include modifications in diagnostic approaches that are less risky. ${ }^{13}$ It is unknown, however, how noninvolved clinicians react to similar cases and whether group knowledge of the event improves the care of future patients. ${ }^{14}$ Understanding how clinicians react to these rare cases can influence the way a region prepares healthcare providers for the possibility of RMSF (and other rare diseases) and may potentially improve the quality of care of patients who present with signs and symptoms of suspected RMSF.

In 2014, a pediatric patient (referred herein as the index case) presented to our institution's pediatric intensive care unit with severe presumed RMSF. Despite aggressive therapy, the patient died of this illness. The primary objectives of the present study are to examine the diagnostic and treatment practices in a nonendemic area before and after a severe pediatric case of RMSF. We hypothesize that inpatient diagnostic testing frequency and initiation of empiric treatment will increase after the occurrence of a severe case of presumed RMSF.

\section{Methods}

This was a retrospective study of pediatric patients seen in the emergency department or admitted to an inpatient service in Penn State Hershey Children's Hospital who were tested for RMSF. Penn State Hershey Children's Hospital is a tertiary care facility located in the central Pennsylvania area. Using the Cerner hospital database, we identified and included the following patients: 1) pediatric patients aged $<18 \mathrm{y}$; 2) patients serologically tested for RMSF via indirect immunofluorescence (our primary mode of diagnostic testing); and 3) patients admitted to the Penn State Hershey Children's Hospital or seen in our emergency department. After we obtained this list of patients, we used our electronic health record to perform a chart review of each case. Data collected included demographics (age, sex, race), timing of RMSF testing, presence of RMSF diagnosis, timing of doxycycline administration (with appropriate administration considered to be within $3 \mathrm{~h}$ of suspicion of RMSF), patient outcome, length of stay, primary clinical setting during evaluation (inpatient or emergency department), and identification, timing, and description of a severe case of confirmed RMSF. ${ }^{15}$ Retrospective review of the patients' medical records was completed between January 1, 2011 and December 31, 2019.

We divided the study population into 2 groups: preindex and postindex. Preindex was defined as cases in which patients were evaluated for RMSF before and including August 15, 2014, the day on which a pediatric patient with a severe case of presumed RMSF died. Postindex was defined as the cases after, but not including, this event.

The study was reviewed by the Penn State College of Medicine institutional review board and determined to be exempt from institutional review board oversight (STUDY00013124). Summary statistics using median, interquartile range, or proportions were reported for clinical and demographic characteristics of the preindex and postindex patients who were tested for RMSF. Fisher's exact test was applied to compare the proportions before and after the index case. Wilcoxon rank sum test with continuity correction was applied to compare the annual incidences between preindex and postindex periods. Analysis was performed using R v4.0.0. $P$ values $<0.05$ were regarded as statistically significant.

\section{Results}

During the study period, an average of $8829 \pm 2138$ pediatric patients (age $0-18$ y) were seen in the emergency department per year. A total of 54 patients were included in this study. The age (median [25th percentile, 75th percentile]) of patients evaluated was $14.5(8.6,16) \mathrm{y}$ preindex and $8.3(3.6,14)$ y postindex. Seven preindex patients $(50 \%)$ were male, and $27(68 \%)$ postindex were male. Other patient characteristics were similar (Table 1).

The index case patient was a 3-y-old female who presented to an outside hospital with nonspecific upper respiratory infection symptoms, rash, and encephalopathy during the summer of 2014. There was no history of travel, but there was a history of a dog tick bite. The patient was transferred to our institution's pediatric intensive care unit, where she was empirically started on broad-spectrum antibiotics including doxycycline within $95 \mathrm{~min}$. The patient developed progressive encephalopathy, acute respiratory failure secondary to acute respiratory distress syndrome, and profound shock. RMSF testing detected no significant level of Rickettsia rickettsii IgG antibody. Based on the findings of fever and generalized rash that began on the wrists and ankles and 
Table 1. Demographic characteristics

\begin{tabular}{lcc}
\hline Patient characteristics & $\begin{array}{c}\text { Preindex case } \\
\text { (including } \\
\text { index case) }\end{array}$ & Postindex case \\
\hline Age, median $\left(25^{\text {th }}, 75^{\text {th }}\right.$ & $14.5(8.6,16)$ & $8.3(3.6,14)$ \\
percentile), & & \\
Race, n (\%) & & \\
White & $12(86 \%)$ & $27(68 \%)$ \\
Asian & $1(7 \%)$ & $1(3 \%)$ \\
African American & $1(7 \%)$ & $3(8 \%)$ \\
Other & $0(0 \%)$ & $9(23 \%)$ \\
Sex, n $(\%)$ & $7(50 \%)$ & $27(68 \%)$ \\
Male & $7(50 \%)$ & $13(33 \%)$ \\
Female & $4.2(1.7,12)$ & $5.0(2.8,9.6)$ \\
Length of stay, median & & \\
(25 ${ }^{\text {th }}, 75^{\text {th }}$ percentile), d & & \\
Outcome, n $(\%)$ & $13(93 \%)$ & $40(100 \%)$ \\
Survived & $1(7 \%)$ & $0(0 \%)$ \\
Died & $1(7 \%)$ & $1(3 \%)$ \\
RMSF diagnosed, n $(\%)$ & $1(100 \%)$ & $0(0 \%)$ \\
Died & $12(86 \%)$ & $36(90 \%)$ \\
Location, n (\%) & $2(14 \%)$ & $4(10 \%)$ \\
Inpatient & & \\
Emergency & & \\
\hline
\end{tabular}

then spread centrally to involve the legs, buttocks, trunk, and face (with later stages becoming petechial), as well as pediatric infectious diseases consultation, the patient's condition was determined to be consistent with RMSF. After $3 \mathrm{~d}$ in the hospital, the patient died of presumed RMSF. On review of the electronic health record, the patient was evaluated $4 \mathrm{~d}$ before presentation for a diffuse rash on her feet and fever, diagnosed as a viral exanthem. This was the only pediatric patient with (presumed) RMSF to our knowledge who died during the study period.

Fourteen patients were evaluated for RMSF before the index case. More patients $(\mathrm{n}=40)$ were evaluated annually for RMSF after the index case $(P<0.05)$. Twelve $(86 \%)$ preindex patients received empiric doxycycline during testing, compared to 31 (78\%) postindex patients, but this was not statistically significant $(P=0.70)$. Seven $(50 \%)$ preindex patients were administered doxycycline within 3 $\mathrm{h}$, compared to 21 (53\%) postindex patients, but this was also not statistically significant $(P=1.0)$ (Table 2).

Three preindex children who were tested for RMSF were $<8$ y of age, and all $(100 \%)$ received empiric doxycycline treatment. Twenty postindex children were $<8$ y of age, and $16(80 \%)$ received empiric doxycycline treatment $(P=1.0)$.

Four years after the index case, in 2019, a decrease in empiric and urgent initiation of doxycycline administration was noted in patients tested for RMSF (Figure 1;
Table 3). One case of severe RMSF was diagnosed 4 y after the index case; doxycycline was administered within $3 \mathrm{~h}$ after RMSF testing was performed, and the patient survived.

\section{Discussion}

This study found that after a severe case of RMSF in an area currently considered nonendemic, diagnostic testing for RMSF appropriately increased. Not all patients after this case, however, received empiric treatment during RMSF testing. In cases in which empiric treatment was initiated, there was no difference in the frequency of doxycycline administration within the 3-h time window between preindex and postindex patients. Our findings may represent an underappreciation of RMSF severity and may have implications for the approach to diagnostic and treatment practices in nonendemic RMSF areas.

RMSF is a vector-borne illness. ${ }^{2}$ Therefore, it depends on the presence of ticks and a favorable environment that allows them to thrive. ${ }^{16}$ Warmer weather allows the tick to reproduce and facilitates the presence of human hosts. ${ }^{16}$ This may contribute to tick bites and the spread of RMSF, even if it is only present in a small population of ticks. ${ }^{10,11}$ As temperatures rise across the United States, including Pennsylvania, institutions such as ours need to be prepared to recognize the potential presence of this severe illness, diagnose it, and initiate early treatment to avoid the severe consequences of the disease. ${ }^{17}$

To avoid the morbidity and mortality associated with RMSF, empiric treatment with doxycycline is recommended. ${ }^{2,7}$ In pediatric patients, doxycycline is

Table 2. Inpatient Rocky Mountain spotted fever diagnostic and empiric treatment practices

\begin{tabular}{|c|c|c|c|}
\hline & $\begin{array}{l}\text { Preindex } \\
\text { case }^{a}\end{array}$ & $\begin{array}{l}\text { Postindex } \\
\text { case }\end{array}$ & $\begin{array}{l}P \\
\text { value }\end{array}$ \\
\hline RMSF testing, $\mathrm{n}$ & 14 & 40 & $0.04^{b}$ \\
\hline $\begin{array}{l}\text { Empiric doxycycline } \\
\text { administration, n (\%) }\end{array}$ & $12(86 \%)$ & $31(78 \%)$ & 0.70 \\
\hline $\begin{array}{l}\text { Empiric doxycycline } \\
\text { administration within } 6 \\
\mathrm{~h} \text { in suspected RMSF } \\
\text { cases, } \mathrm{n}(\%)\end{array}$ & $10(71 \%)$ & $28(70 \%)$ & 1.00 \\
\hline $\begin{array}{l}\text { Empiric doxycycline } \\
\text { administration within } 3 \\
\mathrm{~h} \text { in suspected RMSF } \\
\text { cases, } \mathrm{n}(\%)\end{array}$ & $7(50 \%)$ & $21(53 \%)$ & 1.00 \\
\hline
\end{tabular}




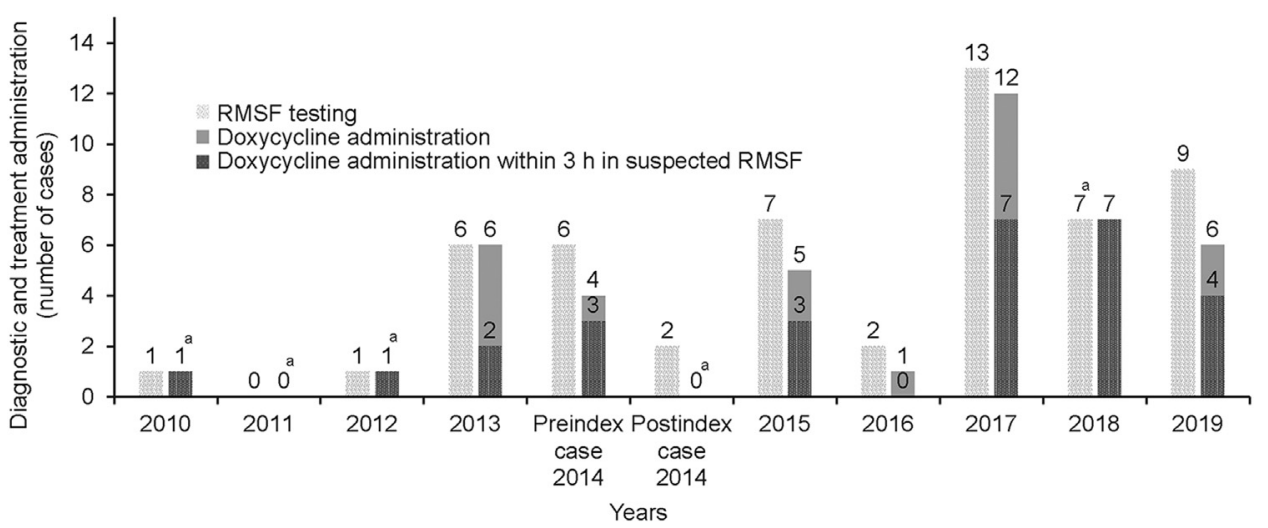

Figure 1. Count of testing and frequency of doxycycline administration (total and within $3 \mathrm{~h}$ ) in patients with suspected Rocky Mountain spotted fever. ${ }^{a}$ Counts of total doxycycline administration and administration within $3 \mathrm{~h}$ were equal.

associated with adverse effects, particularly dental staining. ${ }^{18}$ Recent American Academy of Pediatrics (AAP) guidelines, however, strongly indicate that the risks of this outweigh the benefits of treatment. ${ }^{18}$ Thus, in cases inw hich RMSF is suspected, the patient should be tested, started on doxycycline within $5 \mathrm{~d}$ of symptom onset, and should continue treatment until the results return.

Our study found that despite AAP recommendations, not all patients received empiric treatment, including patients $<8$ y of age after the index case. This is consistent with previous studies examining RMSF therapeutic practices. Although a majority of providers identified doxycycline as the appropriate antimicrobial through a survey, only $35 \%$ chose this antibiotic in children $<8$ y of age. ${ }^{19}$ In Tennessee, an endemic area, it was reported that a high proportion of survey participants were unaware that doxycycline is the treatment of choice for children $<8 \mathrm{y}$ of age. ${ }^{20}$ Another study reported that treatment delay may be due to a likely decreased awareness of RMSF and reluctance to use doxycycline in children. ${ }^{21}$ Our study confirms that this provider-based issue continues to occur. In addition, this study highlights that education efforts are needed to enhance provider awareness that the clinical decision-making process could contribute to a rare but preventable cause of pediatric mortality.

This present study set a time point of $3 \mathrm{~h}$ after doxycycline administration as appropriate empiric treatment. This may be considered overly conservative, especially because patients with RMSF have a time window of $5 \mathrm{~d}^{2}$ However, when this time point was expanded to $6 \mathrm{~h}$ (to evaluate whether any clinicians recognized the possibility of RMSF at a later point), there were still patients who did not receive empiric treatment. In endemic areas, owing to the disease's nonspecific symptomology, treatment is often initiated even if an alternative diagnosis is more likely. ${ }^{2}$ In our area, however, RMSF is nonendemic; thus, it may not be considered as a high-priority diagnosis. Nevertheless, because of the severity of

Table 3. Inpatient Rocky Mountain spotted fever diagnostic and empiric treatment practices divided by year

\begin{tabular}{lclll}
\hline & RMSF testing $(n)$ & $\begin{array}{l}\text { Doxycycline } \\
\text { administration in } \\
\text { suspected RMSF (n, \%) }\end{array}$ & $\begin{array}{l}\text { Doxycycline } \\
\text { administration within } \\
6 \text { h in suspected } \\
\text { RMSF (n, \%) }\end{array}$ & $\begin{array}{l}\text { Doxycycline } \\
\text { administration within } 3 \text { h } \\
\text { in suspected RMSF (n, \%) }\end{array}$ \\
\hline 2010 & 1 & $1(100 \%)$ & $0(10 \%)$ & $1(100 \%)$ \\
2011 & 0 & $0(0 \%)$ & $1(100 \%)$ & $0(0 \%)$ \\
2012 & 1 & $1(100 \%)$ & $2(33 \%)$ & $2(33 \%)$ \\
2013 & 6 & $6(100 \%)$ & $4(67 \%)$ & $3(50 \%)$ \\
Preindex case 2014 & 6 & $4(67 \%)$ & $0(0 \%)$ & $0(0 \%)$ \\
Postindex case 2014 & 2 & $0(0 \%)$ & $5(71 \%)$ & $3(43 \%)$ \\
2015 & 7 & $5(71 \%)$ & $1(50 \%)$ & $0(0 \%)$ \\
2016 & 2 & $1(50 \%)$ & $12(92 \%)$ & $7(54 \%)$ \\
2017 & 13 & $12(92 \%)$ & $7(100 \%)$ & $7(100 \%)$ \\
2018 & 7 & $7(100 \%)$ & $5(56 \%)$ & $4(44 \%)$ \\
2019 & 9 & $6(67 \%)$ & & 0 \\
\hline
\end{tabular}

${ }^{a}$ Includes index case. 
RMSF, we argue that if a clinician considers this a possible diagnosis, there likely was a delay in that consideration; thus, this conservative approach should be taken to avoid potential complications. In addition, despite studies showing that treatment for RMSF should be initiated within $5 \mathrm{~d}$ of onset of symptoms, initiation of treatment as soon as possible when this disease is suspected is supported. $^{2}$

This study is unique in that we report how our practices changed after the RMSF index case. Because our institution is in an academic center, this case did undergo routine morbidity and mortality conference review focusing on inpatient care (not outpatient care); furthermore, due to the rarity of this diagnosis, it was presented in resident academic conferences. The index case provided a real-life example of the lethality of RMSF, the ease with which the diagnosis was overlooked, and how it should appropriately have resulted in a change in practice (even among noninvolved clinicians). The only significant practice change that was noted, however, was an increase in testing (with only $1 \mathrm{y}$ in which all patients tested received empiric treatment). RMSF immunofluorescence assay (IFA) testing costs approximately $\$ 27$ USD. ${ }^{22}$ At our institution, however, the test is analyzed externally, and the turnaround time can be 2 to $3 \mathrm{~d} .^{23}$ Thus, although the index of suspicion appropriately increased, definitive action to prevent harm was not undertaken because results are not immediately available and diagnostic testing depends on the patient's antibody response (a negative test does not necessarily mean the patient does not have RMSF).

Various factors likely contributed to the approach our clinicians took to patients with suspected RMSF. First, the AAP guidelines for doxycycline administration in suspected RMSF are relatively recent; thus, not all clinicians may be aware of this new recommendation, especially because clinicians are taught early in their training to avoid doxycycline use in children $<8 \mathrm{y}$ of age. Because RMSF is rare in our region, even if clinicians do consider RMSF, it may be considered unlikely, thus decreasing the priority of the timeliness of doxycycline administration. Clinicians may have collected a thorough history and determined that although the patient has symptoms similar to RMSF, the risk factors are likely lower (ie, the patient did not have any recent travel history or tick bites or did not frequent any wooded regions). Finally, most of these children presented in a nonacute fashion (ie, were not in shock, respiratory failure, or encephalopathic, like our index case). Thus, the relatively healthy condition of the patient affected how the clinician ordered treatment (urgently versus not). Based on our study findings, known microbiology testing limitations, and the potential for patient harm in unrecognized cases, we recommend that empiric doxycycline should be administered if a provider suspects or tests for RMSF.
It is unclear why there was only $1 \mathrm{y}$ (2018) in which all patients tested for RMSF received empiric treatment, with a potential decreasing trend in RMSF testing the year thereafter. Possibilities include faculty and resident turnover; the clinicians involved in the case may have no longer been present within the institution (thereby resulting in a collective decrease in the index of suspicion). The personal impact of the event may have diminished with the passage of time. Less experienced clinicians may be present. Finally, this case may no longer be discussed within the residency education curriculum.

There were various limitations in this study. This was a single-center retrospective study with a small sample size. Due to limitations in data retrieval from our electronic health record, variation in provider documentation practices, and potential for omitted data, we focused only on patients who were tested for RMSF rather than those who should have been considered. Thus, we did not review patients who may have been empirically treated for RMSF without testing or who may have had symptoms possibly associated with RMSF. We did not determine whether sepsis-associated organ dysfunction was present. The index case was seen initially in the outpatient setting. Therefore, it is unknown whether a peer review discussion took place after this case or if there was an impact on diagnostic or treatment practices in the outpatient setting. Finally, it is unknown whether the practice changes we have described were truly in response to the index case or to the rise in RMSF cases noted nationwide.

\section{Conclusions}

Our study found that inpatient testing for RMSF increased after the index case, but not all patients received empiric treatment. This may represent an underappreciation of RMSF severity in light of a devastating case that should have triggered a change in practice, even among noninvolved clinicians. We suggest that when severe, rare, but possibly treatable diseases such as RMSF occur, all clinicians should be educated on the diagnostic and treatment approach to reduce the risk of morbidity and mortality.

Author Contributions: Study concept and design (CK, GDC, NJT); acquisition of the data (CK); analysis of the data (CK, SZ); drafting of the manuscript (CK); critical revision of the manuscript (GDC, SZ, NJT); approval of the final manuscript (all authors).

Financial/Material Support: The project described was supported by the National Center for Advancing Translational Sciences, National Institutes of Health, through Grant UL1 TR002014. The content is solely the responsibility of the authors and does not necessarily represent the official views of the National Institutes of Health.

Disclosures: None. 


\section{References}

1. Gottlieb M, Long B, Koyfman A. The evaluation and management of Rocky Mountain spotted fever in the emergency department: a review of the literature. J Emerg Med. 2018;55(1):42-50.

2. Regan JJ, Traeger MS, Humpherys D, Mahoney DL, Martinez M, Emerson GL, et al. Risk factors for fatal outcome from rocky mountain spotted fever in a highly endemic area-Arizona, 2002-2011. Clin Infect Dis. 2015;60(11):1659-66.

3. Buckingham SC, Marshall GS, Schutze GE, Woods CR, Jackson MA, Patterson LE, et al. Clinical and laboratory features, hospital course, and outcome of Rocky Mountain spotted fever in children. $J$ Pediatr. 2007;150(2):180-4.

4. Woods CR. Rocky Mountain spotted fever in children. Pediatr Clin North Am. 2013;60(2):455-70.

5. Allen HC, Welliver Sr RC, Fogarty MW, Gessouroun M, Henry ED. Intravenous immunoglobulin therapy for cerebral vasculitis associated with Rocky Mountain spotted fever. J Pediatr Intensive Care. 2017;6(2):142-4.

6. Sekeyová Z, Danchenko M, Filipčík P, Fournier PE. Rickettsial infections of the central nervous system. PLoS Negl Trop Dis. 2019;13(8):e0007469.

7. Kirkland KB, Wilkinson WE, Sexton DJ. Therapeutic delay and mortality in cases of Rocky Mountain spotted fever. Clin Infect Dis. 1995;20(5):1118-21.

8. O'Reilly M, Paddock C, Elchos B, Goddard J, Childs J, Currie M. Physician knowledge of the diagnosis and management of Rocky Mountain spotted fever: Mississippi, 2002. Ann N Y Acad Sci. 2003;990:295-301.

9. Tull R, Ahn C, Daniel A, Yosipovitch G, Strowd LC. Retrospective study of Rocky Mountain spotted fever in children. Pediatr Dermatol. 2017;34(2):119-23.

10. Parola P, Socolovschi C, Jeanjean L, Bitam I, Fournier P-E, Sotto A, et al. Warmer weather linked to tick attack and emergence of severe rickettsioses. PLoS Negl Trop Dis. 2008;2(11):e338.

11. Daniel M, Danielová V, Fialová A, Malý M, Kř́ǔ B, Nuttall PA. Increased relative risk of tick-borne encephalitis in warmer weather. Front Cell Infect Microbiol. 2018;8:90.

12. Eremeeva ME, Dasch GA. Challenges posed by tick-borne rickettsiae: eco-epidemiology and public health implications. Front Public Health. 2015;3:55.
13. Heyhoe J, Birks Y, Harrison R, O’Hara JK, Cracknell A, Lawton R. The role of emotion in patient safety: are we brave enough to scratch beneath the surface? J R Soc Med. 2016;109(2):52-8.

14. Chuang Y-T, Ginsburg L, Berta WB. Learning from preventable adverse events in health care organizations: development of a multilevel model of learning and propositions. Health Care Manage Rev. 2007;32(4):330-40.

15. Weiss SL, Peters MJ, Alhazzani W, Agus MSD, Flori HR, Inwald DP, et al. Surviving sepsis campaign international guidelines for the management of septic shock and sepsisassociated organ dysfunction in children. Pediatr Crit Care Med. 2020;21(2):e52-106.

16. Bouchard C, Dibernardo A, Koffi J, Wood H, Leighton P, Lindsay L. N increased risk of tick-borne diseases with climate and environmental changes. Can Commun Dis Rep. 2019;45(4):83-9.

17. Liu HH, Cushinotto L, Giger O, Daum G, McBride P, Negron EA, et al. Increasing Babesiosis in southeastern Pennsylvania, 2008-2017. Open Forum Infect Dis. 2019;6(3):ofz066.

18. Todd SR, Dahlgren FS, Traeger MS, Beltrán-Aguilar ED, Marianos DW, Hamilton C, et al. No visible dental staining in children treated with doxycycline for suspected Rocky Mountain spotted fever. J Pediatr. 2015;166(5):1246-51.

19. Zientek J, Dahlgren FS, McQuiston JH, Regan J. Self-reported treatment practices by healthcare providers could lead to death from Rocky Mountain spotted fever. J Pediatr. 2014;164(2):416-8.

20. Mosites E, Carpenter LR, McElroy K, Lancaster MJ, Ngo TH, McQuiston J, et al. Knowledge, attitudes, and practices regarding Rocky Mountain spotted fever among healthcare providers, Tennessee, 2009. Am J Trop Med Hyg. 2013;88(1):162-6.

21. Hayden AM, Marshall GS. Rocky Mountain spotted fever at Koair children's hospital, 1990-2002. J Ky Med Assoc. 2004;102(5):209-14.

22. Connally NP, Hinckley AF, Feldman KA, Kemperman M, Neitzel D, Wee S-B, et al. Testing practices and volume of non-Lyme tickborne diseases in the United States. Ticks Tick Borne Dis. 2016;7(1):193-8.

23. Brzozowski AK, Silk BJ, Berkelman RL, Loveys DA, Caliendo AM. Use, location, and timeliness of clinical microbiology testing in Georgia for select infectious diseases. J Public Health Manag Pract. 2012;18(4):E4-10. 